Article

\title{
Interactive Effects of Glycine Equivalent, Cysteine, and Choline on Growth Performance, Nitrogen Excretion Characteristics, and Plasma Metabolites of Broiler Chickens Using Neural Networks Optimized with Genetic Algorithms
}

\author{
Philipp Hofmann ${ }^{1}$, Wolfgang Siegert ${ }^{1, *}$, Hamed Ahmadi ${ }^{2}{ }^{\circledR}$, Jochen Krieg ${ }^{1}{ }^{\circledR}$, Moritz Novotny ${ }^{1}$, \\ Victor D. Naranjo ${ }^{3}$ (D) and Markus Rodehutscord ${ }^{1}$ (D) \\ 1 Institute of Animal Science, University of Hohenheim, 70599 Stuttgart, Germany; \\ philipp_hofmann@uni-hohenheim.de (P.H.); jochen.krieg@uni-hohenheim.de (J.K.); \\ moritz.novotny@uni-hohenheim.de (M.N.); markus.rodehutscord@uni-hohenheim.de (M.R.) \\ 2 Bioscience and Agriculture Modeling Research Unit, Department of Poultry Science, \\ Tarbiat Modares University, Tehran 14115-336, Iran; hamed.ahmadi@modares.ac.ir \\ 3 Evonik Nutrition \& Care GmbH, 63457 Hanau, Germany; victor.naranjo@evonik.com \\ * Correspondence: inst450@uni-hohenheim.de; Tel.: +49-711-459-22420
}

Received: 6 July 2020; Accepted: 7 August 2020; Published: 11 August 2020

Simple Summary: The negative effects of nitrogen emissions caused by animal husbandry on the environment can be reduced by lowering the crude protein content in the diets of broiler chickens. The nonessential amino acids glycine and serine, investigated together as glycine equivalent, can limit the growth of broiler chickens fed diets that are low in crude protein. The response of broiler chickens to dietary glycine equivalent is not constant and is affected by endogenous precursors of glycine equivalent and metabolic processes that dissipate glycine equivalent. Choline can be converted to glycine, and glycine equivalent is required to form cysteine from methionine. The present study investigated interactive effects among dietary glycine equivalent, cysteine, and choline in broiler chickens. The results showed that the gain:feed ratio increased with dietary glycine equivalent supplementation. The extent of interactive effects among glycine equivalent, cysteine, and choline on the gain:feed ratio was hardly pronounced. Very high nitrogen-utilization efficiency with low variation among treatments was found. The findings indicate that small differences in nitrogen-utilization efficiency caused low glycine equivalent dissipation for nitrogen excretion, likely resulting in small interactive effects among dietary glycine equivalent, cysteine, and choline. These results contribute to further dietary crude protein reduction in feed for broiler chickens.

Abstract: Responses of broiler chickens to dietary glycine equivalent (Glyequi) are affected by dietary cysteine and choline. Hence, this study investigated interactive effects among dietary Glyequi, cysteine, and choline on the growth of broiler chickens. Male Ross 308 broiler chickens were maintained in 105 metabolism units (10 birds/unit) from days 7 to 22. Excreta were collected in 12-h intervals from days 18 to 21 . Blood was sampled on day 22 (1 bird/unit). Five levels each of Gly equi $(9-21 \mathrm{~g} / \mathrm{kg})$, cysteine $(2-5 \mathrm{~g} / \mathrm{kg})$, and choline $(0.5-1.7 \mathrm{~g} / \mathrm{kg})$ were tested under 15 diets in 7 replicates each following a fractional central composite design. Another diet was provided to five metabolism units (15 birds/unit) to measure prececal amino acid digestibility. Data were evaluated using neural networks. The gain:feed ratio (G:F) increased with digestible Glyequi intake. Differences between low and high digestible cysteine intake were low. Effects of choline intake on G:F were low. Nitrogen-utilization efficiency (NUE) was high $(\geq 77 \%)$, with low variation among treatments. Plasma metabolites varied among treatments and indicated that metabolism of Glyequi, cysteine, 
and choline was influenced. These findings showed that interactive effects of dietary Gly equi, cysteine, and choline on growth were small, possibly because NUE was barely influenced.

Keywords: broiler chickens; interactive effect; glycine equivalent; cysteine; choline; neural networks; nitrogen-utilization efficiency; uric acid; ammonia

\section{Introduction}

The negative impacts of nitrogen $(\mathrm{N})$ emissions from animal husbandry can be diminished by reducing dietary crude protein $(\mathrm{CP})$ in feeds. Very low $\mathrm{CP}$ diets are often accompanied by decreased growth in broiler chickens when the nonessential amino acids (AA) glycine (Gly) and serine (Ser) — considered together as glycine equivalent (Gly equi) —are not adequately supplied because these AA can induce a growth limiting effect [1-3]. The Glyequi requirement is not constant, with dietary Glyequi concentrations varying from below 10 to above $16 \mathrm{~g} / \mathrm{kg}$ among studies to achieve $95 \%$ of the maximum gain:feed ratio (G:F) [4]. These variations were explained by different amounts of

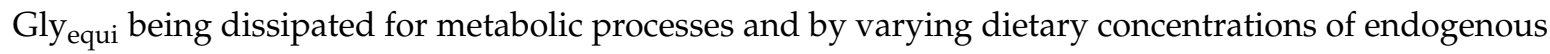
precursors of Gly and Ser.

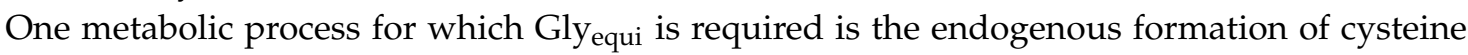
(Cys) from methionine (Met). Homocysteine (Hcy) and cystathionine (Cysta) are intermediates in this metabolic conversion, and Ser is required to form Cysta from Hcy [5]. This was suggested as one explanation for the interactive effects between dietary Glyequi and Cys on growth found in a previous meta-analysis [6]. The extent of these interactive effects cannot be explained only by the formation of Cys from Met. Further influences must have been responsible for the extent of interactive effects between dietary Glyequi and Cys on growth found in the meta-analysis by Siegert et al. [6].

Choline (Cho) is an endogenous precursor of Gly in the metabolic pathway involving the conversion of Cho $\rightarrow$ betaine $\rightarrow$ dimethylglycine $\rightarrow$ sarcosine $\rightarrow$ Gly [7]. The conversion of Cho to Gly is connected to Cys formation from Met and can only occur when one methyl group of betaine is transferred to Hcy by betaine-Hcy-methyltransferase (BHMT; EC 2.1.1.5) to form Met [8]. Concurrently, Met can be synthesized by transferring the methyl groups of dimethylglycine and sarcosine via the folate pool to Hcy by Met-synthase (MS; EC 2.1.1.13) [9]. Interactive effects between Gly equi and Cho on growth have been described previously; however, the extent of these interactive effects cannot alone be explained by the endogenous conversion of Cho to Gly [10]. Therefore, varying dietary Cys concentrations that affect the conversion of Hcy to Met could influence the extent of interactive effects between Glyequi and Cho on growth. Further, Cho as a methyl group donor and precursor of Gly might provide a further explanation regarding the interactive effects of Gly equi and Cys on growth as observed in the meta-analysis by Siegert et al. [6].

The formation of uric acid (UA) is a Gly equi-dissipating process because one molecule of Gly is required to form the purine ring of UA [11]. An increase in nitrogen-utilization efficiency (NUE) leads


Therefore, varying NUE influences the response to dietary Gly equi and likely affects the interactive effects among Gly equi, Cys, and Cho on growth.

We hypothesized that investigating different dietary concentrations of Glyequi, Cys, and Cho together could explain the interactive effects between Gly equi and Cys and between Gly equi and Cho that have previously been found in other studies. Therefore, we investigated the interactive effects among dietary Glyequi, Cys, and Cho in a growth study. Digestibility was analyzed to determine the prececal digestible AA intake. Total $\mathrm{N}$ excretion was measured to determine NUE, and plasma metabolites were analyzed as indicators of metabolism of Gly ${ }_{\text {equi }}$, Cys, and Cho. Neural networks optimized with genetic algorithms were used for data evaluation. 


\section{Materials and Methods}

\subsection{Experimental Design}

Two trials were run simultaneously. All dietary treatments were randomly distributed in a completely randomized block design.

A growth trial was conducted to investigate the interactive effects among dietary Gly equi, Cys, and $\mathrm{Cho}$ at five concentrations on growth, total $\mathrm{N}$ excretion, and plasma metabolites. A fractional central composite design [12] was used. This resulted in 15 dietary treatments that were tested in seven replicates each.

A digestibility trial was undertaken to determine the prececal AA digestibility of one diet in five replicates. This was done to obtain the prececal digestible AA concentrations of diets used in the growth trial.

\subsection{Birds and Housing}

The experiment was performed at the Agricultural Experiment Station of the University of Hohenheim, Germany. It was approved by the Regierungspräsidium Tübingen, Germany (Project no. $\mathrm{HOH} 48 / 17 \mathrm{TE}$ ), and was conducted according to the German Animal Welfare Legislation. Male Ross 308 broiler hatchlings were delivered by a commercial hatchery (Brüterei Süd ZN der BWE-Brüterei Weser-Ems GmbH \& Co. KG, Regenstauf, Germany). Birds were held in five floor pens $(3 \mathrm{~m} \times 4 \mathrm{~m})$ on dedusted wood shavings and received a commercial starter diet containing $215 \mathrm{~g} \mathrm{CP} / \mathrm{kg}$ and 12.5 MJ ME/kg (415002025 Club Mastkükenstarter, Deutsche Tiernahrung Cremer GmbH \& Co. KG, Mannheim, Germany) up to day 7. Then, 1125 birds were distributed to metabolism units and placed on a mesh-wired floor. The birds were allocated to the metabolism units so that an equal mean bird weight was achieved in every unit of both the trials. Feed and water were provided for ad libitum consumption throughout the experiment. Lighting was continuous during the first 3 days after placement, followed by 18-h light and 6-h dark cycle until the end of the experiment. Temperature was maintained at $34^{\circ} \mathrm{C}$ for the first 3 days and was gradually decreased to $26^{\circ} \mathrm{C}$ on the last day of the experiment.

In the growth trial, a total of 1050 broiler chickens were distributed to $105(1 \mathrm{~m} \times 1 \mathrm{~m} \times 1 \mathrm{~m})$ metabolism units. Each diet was tested in 7 metabolism units and each metabolism unit contained 10 birds. The experimental diets were fed from days 7 to 22 .

In the digestibility trial, 15 out of 75 birds were assigned to one of five $(2 \mathrm{~m} \times 1 \mathrm{~m} \times 1 \mathrm{~m})$ metabolism units. The birds received the experimental diets from days 7 to 21 .

\subsection{Experimental Diets}

The diets were mainly based on corn and casein (Table 1) and were mixed at the certified feed mill of the University of Hohenheim. Concentrations of all nutrients listed herein are based on a standardized dry matter (DM) of $88 \%$, unless otherwise stated. The concentrations of essential AA including Met + Cys were calculated at $105 \%$ of the recommendations of the Gesellschaft für Ernährungsphysiologie [13]. The concentrations of the nonessential AA alanine, aspartic acid/asparagine, and glutamic acid/glutamine were calculated at 8.6, 15.4, and $28.1 \mathrm{~g} / \mathrm{kg}$, respectively, because lower concentrations of these AA cause limited growth [14]. Concentrations of AA were adjusted by adding AA in free form. Other nutrients were formulated to meet or exceed the recommendations of the Gesellschaft für Ernährungsphysiologie [13]. 
Table 1. Composition of the experimental diets arranged according to the fractional central composite design $(\mathrm{g} / \mathrm{kg})$.

\begin{tabular}{|c|c|c|c|c|c|c|c|c|c|c|c|c|c|c|c|c|}
\hline \multirow[b]{2}{*}{ Treatment } & \multicolumn{15}{|c|}{ Growth Trial } & \multirow{2}{*}{$\begin{array}{c}\text { Digestibility Trial } \\
\mathbf{P}\end{array}$} \\
\hline & $\mathbf{A}$ & B & $\mathrm{C}$ & D & $\mathrm{E}$ & F & G & $\mathrm{H}$ & I & $\mathrm{J}$ & K & $\mathbf{L}$ & $\mathbf{M}$ & $\mathbf{N}$ & $\mathrm{O}$ & \\
\hline & \multicolumn{16}{|c|}{ Non-varying ingredients } \\
\hline Corn & \multicolumn{16}{|c|}{794} \\
\hline Casein & \multicolumn{16}{|c|}{84} \\
\hline Soybean oil & \multicolumn{16}{|c|}{30} \\
\hline L-Arginine & \multicolumn{16}{|c|}{7.2} \\
\hline L-Aspartic acid & \multicolumn{16}{|c|}{6.5} \\
\hline L-Lysine $\cdot \mathrm{HCl}$ & \multirow{2}{*}{\multicolumn{16}{|c|}{$\begin{array}{l}4.8 \\
3.8\end{array}$}} \\
\hline L-Valine & & \multicolumn{15}{|c|}{3.8} \\
\hline L-Threonine & \multicolumn{16}{|c|}{2.6} \\
\hline L-Alanine & \multicolumn{16}{|c|}{2.2} \\
\hline L-Isoleucine & \multicolumn{16}{|c|}{2.2} \\
\hline L-Glutamic acid & \multicolumn{16}{|c|}{1.8} \\
\hline L-Phenylalanine & \multicolumn{16}{|c|}{$\begin{array}{l}1.1 \\
0.4\end{array}$} \\
\hline L-Tryptophan & \multicolumn{16}{|c|}{0.4} \\
\hline L-Tyrosine & \multirow{2}{*}{\multicolumn{16}{|c|}{$\begin{array}{c}0.2 \\
15.3\end{array}$}} \\
\hline Limestone & & & & & & & & & & & & & & & & \\
\hline Monocalcium phosphate & \multicolumn{16}{|c|}{15.0} \\
\hline Sodium bicarbonate & \multicolumn{16}{|c|}{3.3} \\
\hline Vitamin premix ${ }^{1}$ & \multicolumn{16}{|c|}{2.0} \\
\hline Sodium chloride & \multicolumn{16}{|c|}{1.0} \\
\hline Trace element premix ${ }^{2}$ & \multicolumn{16}{|c|}{0.5} \\
\hline & \multicolumn{16}{|c|}{ Varying ingredients } \\
\hline DL-Methionine & 1.7 & 2.5 & 2.5 & 0.8 & 0.8 & 3.4 & 1.7 & 1.7 & 1.7 & - & 2.5 & 2.5 & 0.8 & 0.8 & 1.7 & - \\
\hline $\mathrm{L}-\mathrm{C} y$ steine $\cdot \mathrm{HCl} \cdot \mathrm{H}_{2} \mathrm{O}$ & 3.2 & 1.7 & 1.7 & 4.6 & 4.6 & 0.3 & 3.2 & 3.2 & 3.2 & 6.0 & 1.7 & 1.7 & 4.6 & 4.6 & 3.2 & 0.3 \\
\hline Glycine & 0.2 & 3.4 & 3.4 & 3.4 & 3.4 & 6.6 & 6.6 & 6.6 & 6.6 & 6.6 & 9.7 & 9.7 & 9.7 & 9.7 & 12.9 & 0.2 \\
\hline Choline chloride $^{3}$ & 2.6 & 1.6 & 3.7 & 1.6 & 3.7 & 2.6 & 0.5 & 2.6 & 4.7 & 2.6 & 1.6 & 3.7 & 1.6 & 3.7 & 2.6 & 2.6 \\
\hline Cornstarch & 14.4 & 12.9 & 10.8 & 11.7 & 9.6 & 9.2 & 10.1 & 8.0 & 5.9 & 6.9 & 6.6 & 4.5 & 5.4 & 3.3 & 1.7 & 14.0 \\
\hline $\mathrm{TiO}_{2}$ & - & - & - & - & - & - & - & - & - & - & - & - & - & - & - & 5.0 \\
\hline
\end{tabular}

1 Vitamin premix (Miavit GmbH, Essen, Germany) provided per kg of diet: 10,000 IU vitamin A as retinyl acetate (3a672a); 3000 IU vitamin D3 as cholecalciferol (E671); 30 IU vitamin $\mathrm{E}$ as all rac- $\alpha$-tocopherol (3a700); $2.4 \mathrm{mg}$ vitamin K3 as menadione (3a711); $100 \mu \mathrm{g}$ biotin (3a880); $1 \mathrm{mg}$ folic acid (3a316); $3 \mathrm{mg}$ thiamine (3a821); $6 \mathrm{mg}$ riboflavin; $6 \mathrm{mg}$ pyridoxine (3a831); $30 \mu \mathrm{g}$ vitamin B12; $50 \mathrm{mg}$ nicotinamide (3a315); $14 \mathrm{mg}$ calcium D-pantothenate (3a841). ${ }^{2}$ Trace element premix (Gelamin Gesellschaft für Tierernährung mbH, Memmingen, Germany) provided per $\mathrm{kg}$ of diet: $25 \mathrm{mg}$ calcium from calcium carbonate; $80 \mathrm{mg}$ manganese from manganese-(II)-oxide; $60 \mathrm{mg}$ zinc from zinc-sulfate; $25 \mathrm{mg}$ iron from ferrous-(II)-sulfate monohydrate; $7.5 \mathrm{mg}$ copper from cupric-(II)-sulfate pentahydrate; $0.6 \mathrm{mg}$ iodine from calcium iodate; $0.2 \mathrm{mg}$ selenium from sodium selenite; $15 \mathrm{mg}$ sepiolite. ${ }^{3} 50 \%$ Choline chloride,

$373 \mathrm{~g} / \mathrm{kg}$ choline concentration. 
In the growth trial, different concentrations of dietary Gly equi $(9,12,15,18$, and $21 \mathrm{~g} / \mathrm{kg})$, Cys (2.0, $2.75,3.5,4.25$, and $5.0 \mathrm{~g} / \mathrm{kg}$ ), and Cho $(0.5,0.8,1.1,1.4$, and $1.7 \mathrm{~g} / \mathrm{kg}$ ) (Table 2) were obtained by adding free Gly, free L-Cys $\cdot \mathrm{HCl} \cdot \mathrm{H}_{2} \mathrm{O}$, and choline chloride in variable proportions to a basal mixture. Free DL-Met inclusions were altered to ensure constant Met + Cys concentrations among treatments. Cornstarch was used to compensate for mass differences. Glyequi concentrations used in this study were similar to those that were shown to influence growth in the literature [4]. Cho concentrations were calculated according to those reported by Siegert et al. [10], and Cys concentrations were calculated to include the range of Cys concentrations reported in the meta-analysis by Siegert et al. [6].

Table 2. Combinations of different nutrients and calculated and analyzed nutrient concentrations $(\mathrm{g} / \mathrm{kg})$ of glycine equivalent (Gly equi), cysteine (Cys), and choline (Cho) in the experimental diets.

\begin{tabular}{|c|c|c|c|c|c|c|c|c|c|}
\hline \multirow[t]{2}{*}{ Treatment } & \multicolumn{3}{|c|}{ Level Combinations ${ }^{1}$} & \multicolumn{3}{|c|}{$\begin{array}{l}\text { Calculated Nutrient } \\
\text { Concentrations }\end{array}$} & \multicolumn{3}{|c|}{$\begin{array}{l}\text { Analyzed Nutrient } \\
\text { Concentrations }\end{array}$} \\
\hline & Glyequi & Cys & Cho & Gly equi & Cys & $\mathrm{Cho}^{2}$ & Gly $_{\text {equi }}$ & Cys & $\mathrm{Cho}^{3}$ \\
\hline \multicolumn{10}{|l|}{ Growth trial } \\
\hline A & -2 & 0 & 0 & 9 & 3.5 & 1.1 & 9.1 & 3.5 & n. a. \\
\hline B & -1 & -1 & -1 & 12 & 2.75 & 0.8 & 12.2 & 2.6 & n. a. \\
\hline $\mathrm{C}$ & -1 & -1 & 1 & 12 & 2.75 & 1.4 & 12.0 & 2.6 & 1.4 \\
\hline $\mathrm{D}$ & -1 & 1 & -1 & 12 & 4.25 & 0.8 & 12.0 & 4.4 & 1.0 \\
\hline $\mathrm{E}$ & -1 & 1 & 1 & 12 & 4.25 & 1.4 & 12.2 & 4.3 & 1.6 \\
\hline $\mathrm{F}$ & 0 & -2 & 0 & 15 & 2.0 & 1.1 & 14.9 & 1.8 & n. a. \\
\hline G & 0 & 0 & -2 & 15 & 3.5 & 0.5 & 15.1 & 3.4 & 0.8 \\
\hline H (central diet) & 0 & 0 & 0 & 15 & 3.5 & 1.1 & 15.1 & 3.4 & 1.3 \\
\hline $\mathrm{I}$ & 0 & 0 & 2 & 15 & 3.5 & 1.7 & 15.1 & 3.5 & 2.1 \\
\hline $\mathrm{J}$ & 0 & 2 & 0 & 15 & 5.0 & 1.1 & 15.0 & 5.1 & n. a. \\
\hline $\mathrm{K}$ & 1 & -1 & -1 & 18 & 2.75 & 0.8 & 18.1 & 2.7 & 0.9 \\
\hline $\mathrm{L}$ & 1 & -1 & 1 & 18 & 2.75 & 1.4 & 18.2 & 2.7 & 1.5 \\
\hline M & 1 & 1 & -1 & 18 & 4.25 & 0.8 & 18.4 & 4.4 & n. a. \\
\hline $\mathrm{N}$ & 1 & 1 & 1 & 18 & 4.25 & 1.4 & 18.6 & 4.4 & 1.4 \\
\hline $\mathrm{O}$ & 2 & 0 & 0 & 21 & 3.5 & 1.1 & 21.3 & 3.6 & 1.3 \\
\hline \multicolumn{10}{|l|}{ Digestibility trial } \\
\hline $\mathrm{P}$ & -2 & -2 & 0 & 9 & 2.0 & 1.1 & 8.9 & 1.8 & n. a. \\
\hline
\end{tabular}

${ }^{1}$ According to the fractional central composite design for treatments A-O; ${ }^{2}$ Calculated concentrations were used for further calculations; ${ }^{3}$ n. a. = not analyzed.

The diet fed in the digestibility trial was mixed using the same basal mixture utilized for the growth trial. The concentrations of dietary Gly equi, Cys, and Met were equal to the lowest concentrations of these nutrients in the growth trial. $\mathrm{TiO}_{2}$ was added as an indigestible marker.

One basal mixture that represented approximately $98 \%$ of the final diets of both trials was established, divided into 16 parts, and complemented with the ingredients required for the final diets. The diets were pelleted without using steam through a 3-mm die. The calculated AA concentrations were confirmed by analyses. The variation of the analyzed AA (apart from Glyequi, Cys, and Met) was low among the treatments (Table 2 and Table S1). Dietary CP ranged between 172 and $185 \mathrm{~g} / \mathrm{kg}$. The analyzed Cho concentrations were within the 15\% range of measurement accuracy, thus confirming the calculated values. Calculated dietary Cho concentrations were used for subsequent calculations.

\subsection{Experimental Procedures}

In the growth trial, total bird weight and feed consumption in each metabolism unit were recorded on days 7, 18, and 21 of the experiment to determine the mortality-corrected average daily gain (ADG), average daily feed intake (ADFI), and G:F. Feed intake was measured on a DM basis by determining the DM content of each diet on the same days. Total excreta were collected at 12-h intervals from days 18 to 21 of the experiment. Excreta were carefully cleaned to remove feathers, skin scales, and spilled feed pellets before each collection and then immediately stored at $-20{ }^{\circ} \mathrm{C}$. Spilled feed was picked up throughout the experiment, weighed after being dried, and used for correction of feed DM intake. The excreta of birds collected in four metabolism units were not used for data analysis because of 
errors during excreta collection. The birds were examined at least twice daily. In the case of death, dead birds were considered in the calculation of ADG, ADFI, and G:F. Blood was sampled on day 22 from the brachial vein of one bird per metabolism unit and was placed into a $4 \mathrm{~mL}$ heparinized tube. The bird whose body weight was the closest to the mean body weight of birds in each unit was selected for blood sampling. The feeding regime according to Donsbough et al. [15] described in detail by Hofmann et al. [14] was used before blood sampling. The sample was immediately centrifuged at $1500 \times g$ for $10 \mathrm{~min}$ at $4{ }^{\circ} \mathrm{C}$, and the resulting blood plasma was frozen at $-20{ }^{\circ} \mathrm{C}$.

In the digestibility trial, birds were fed the diet with medium concentrations of Gly equi, Cys, Met, and $\mathrm{Cho}$ (treatment $\mathrm{H}$ in the growth trial) from days 7 to 16 to ensure similar physiological development of the birds in the two trials. Subsequently, they received diet $\mathrm{P}$ (with the lowest concentrations of Gly $_{\text {equi, }}$ Cys, and Met). On day 21, the birds were stunned with a gas mixture of $35 \% \mathrm{CO}_{2}, 35 \% \mathrm{~N}_{2}$, and $30 \% \mathrm{O}_{2}$ and euthanized with $\mathrm{CO}_{2}$ exposure. Digesta was collected from the distal two-thirds of the section between diverticulum vitellinum and $2 \mathrm{~cm}$ anterior to the ileo-ceco-colonic junction by flushing with cold, double-distilled water. Digesta of the 15 birds in each metabolism unit was pooled and immediately frozen at $-20{ }^{\circ} \mathrm{C}$.

\subsection{Chemical Analyses}

Diet $\mathrm{H}$ was ground using a centrifugal mill (ZM 200; Retsch GmbH, Haan, Germany) through a $0.5 \mathrm{~mm}$ sieve to analyze it for crude ash (method no. 8.1), crude fat (method no. 5.1.1), crude fiber (method no. 6.1.1), DM (method no. 3.1), and starch (method no. 7.2.1) according to the official methods for nutrient analyses in Germany [16]. A vibrating cup mill (Pulverisette 9; Fritsch GmbH, Idar-Oberstein, Germany) was used to pulverize all diets and freeze-dried digesta and excreta for AA, CP, Ti, and UA analyses. CP concentration was determined using the Dumas method. The official method 994.12 of the Association of Official Analytical Chemists [17] was used for AA analysis, with minor laboratory adaptions described by Siegert et al. [10]. CP and AA were analyzed by Evonik Nutrition \& Care GmbH (Hanau, Germany). Ti was analyzed as described previously [18]. Cho was analyzed using the method outlined by Siegert et al. [10] by LUFA-ITL GmbH (Kiel, Germany). At least one diet per Cho concentration was analyzed to confirm the values calculated during diet formulation. Accurate quantification of Cho concentrations in digesta was not possible with a limit of quantification of $0.2 \mathrm{~g} / \mathrm{kg}$ DM; therefore, the digestibility of Cho was not determined. The preparation of excreta samples and determination of $\mathrm{DM}, \mathrm{N}$, ammonia $\left(\mathrm{NH}_{3}\right)$, and $\mathrm{UA}$ in the excreta were conducted according to the method described by Hofmann et al. [14]. One milliliter of each blood plasma sample was freeze-dried before being analyzed for free AA and biogenic amines by Evonik Nutrition \& Care $\mathrm{GmbH}$ (Hanau, Germany). Freeze-dried blood plasma was resolubilized with lithium citrate buffer solution and peptides and proteins were precipitated using sulfosalicylic acid. AA were separated using the Biochrom 30 AA analyzer (Biochrom Ltd., Cambridge, UK). The separated AA were then mixed with ninhydrin and quantified photometrically. Blood plasma biogenic amines were determined using high-performance liquid chromatography with fluorescence detection after pre-column derivatization with dansyl chloride and liquid-liquid extraction with methyl tert-butyl ether. Measured homocystine (Hcys) was used to conclude on Hcy concentrations.

\subsection{Calculations}

The following equations were used to calculate $\mathrm{N}$ accretion and NUE:

$$
\begin{aligned}
& \mathrm{N} \text { accretion }(\mathrm{g} / \mathrm{d})=\mathrm{N} \text { intake }(\mathrm{g} / \text { day })-\text { total } \mathrm{N} \text { excretion }(\mathrm{g} / \text { day }) \\
& \mathrm{NUE}(\%)=\mathrm{N} \text { accretion }(\mathrm{g} / \text { day }) / \mathrm{N} \text { intake }(\mathrm{g} / \text { day }) \times 100
\end{aligned}
$$

The uric acid-nitrogen/(uric acid-nitrogen + ammonia-nitrogen) [UA-N/(UA-N $\left.\left.+\mathrm{NH}_{3}-\mathrm{N}\right)\right]$ ratio in the excreta was calculated because it has been reported to be suitable for interpreting the physiological 
processes of $\mathrm{N}$ metabolism in broiler chickens $[14,19]$. Prececal AA digestibility was calculated using the following equation:

Prececal AA digestibility $(\%)=100-\left[\left(\mathrm{TiO}_{2 \text { Diet }} \times \mathrm{AA}_{\text {Digesta }}\right) /\left(\mathrm{TiO}_{2 \text { Digesta }} \times \mathrm{AA}_{\text {Diet }}\right)\right] \times 100$

where, $\mathrm{AA}_{\text {Diet }}$ and $\mathrm{AA}_{\text {Digesta }}$ are the concentrations of the individual $\mathrm{AA}$ and $\mathrm{TiO}_{2 \text { Diet }}$ and $\mathrm{TiO}_{2 \text { Digesta }}$ are the $\mathrm{TiO}_{2}$ concentrations in the diet and the digesta, respectively. Concentrations of prececal digestible Gly $_{\text {equi }}\left(\mathrm{dGly}_{\text {equi }}\right)$, Cys (dCys), and Met (dMet) in the growth trial diets were determined using digestible AA concentrations determined in the digestibility trial and assuming complete digestibility of free AA. The daily intake of dGlyequi, $\mathrm{dCys}$, dMet, or Cho was measured for each metabolism unit by multiplying the concentrations of dGlyequi, dCys, dMet, or Cho with the respective ADFI.

\subsection{Data Modeling and Optimization}

Supervised, fully connected, feed forward neural networks optimized using genetic algorithms were used for data modeling and optimization using MatLab R2017b (The MathWorks Inc., Natick, MA, USA). Genetic algorithms were used to optimize the neural networks by modifying the number of neurons and their coefficients in the hidden layer to minimize mean squared error (MSE). The training of neural networks initiated with one neuron in the hidden layer. The parameters specifying the genetic algorithm [20] were as follows: initial population of 50, generation number of 1000, mutation rate of 0.1 , and crossover rate of 0.85 . The roulette wheel selection method was used for selecting the elite populations for crossover. An MSE of 0.001 was used as the performance function. Hyperbolic tangent and linear functions were the transfer functions for the hidden and output layers, respectively [21]. The dataset was randomly divided into 70\% training and 30\% testing set for each model. Independent models were developed for each response trait. The evaluation of model performance was based on the accuracy of model predictions in the training, testing, and entire data sets using the coefficient of determination $\left(\mathrm{R}^{2}\right)$ and root MSE. The intake of dGly equi, $\mathrm{dCys}$, and Cho were the independent input variables used for data evaluation because nutrient intake accurately describes the nutrient supply of the birds. The relevance of an input variable to the model performance was determined using the variable total effect (VTE) [22], which represents the contribution of an input variable to the variance of a model output. Higher VTE values indicate a higher influence of input variables on model performance. In addition to defining neural networks, genetic algorithms were utilized to analyze the developed neural network models to find combinations of dGlyequi, $\mathrm{dCys}$, and Cho intake for maximized and minimized responses. Regression analyses were performed to determine the dietary concentrations corresponding to the respective intake of digestible nutrients using GraphPad Prism 5.0 (GraphPad Software Inc., San Diego, CA, USA). Figures were prepared using the same software. According to the fractional composite design, not all level combinations can be shown without extrapolating, and the range where interactions can be interpreted decreased with the deviance from level 0 . Therefore, levels $-1,0$, and +1 were defined as low, medium, and high dCys and Cho intake levels, and ranges of dGly equi intake are presented to be wider than high and low dCys and Cho intake. We decided that the relationships between the measured traits and varying nutrients were too low and were not further described when $\mathrm{R}^{2} \leq 0.50$.

\section{Results}

The initial bird weights among metabolism units ranged between 180 and 191 (standard deviation [SD] 2.7) $\mathrm{g} /$ bird on day 7 and were not significantly different among treatments $(p=0.885)$. The survival rate during the experimental phase was $\geq 96 \%$ and was independent of treatment ( 27 out of 1050 birds died in 11 different treatments in the growth trial and 3 out of 75 birds died in the digestibility trial). 


\subsection{Neural Network Model Characteristics}

The characteristics of models for traits with $\mathrm{R}^{2}>0.50$ that were used for further interpretations are described in Tables 3 and 4, and the corresponding model equations are shown in Table S2. The descriptions of neural network models for traits with $\mathrm{R}^{2} \leq 0.50$ are shown in Table S3. Maximum output values were determined at extrapolated dGlyequi, $\mathrm{dCys}$, and Cho intake levels for all traits. Hence, these combinations are not presented.

\subsection{Prececal Amino Acid Digestibility and Prececal Digestible Nutrient Intake}

Prececal AA digestibility in the digestibility trial was between $81 \%$ for Cys and $96 \%$ for arginine and was higher than $90 \%$ for most AA (Table 5). Prececal digestibility of Gly, Ser, Cys, and Met was $84 \%$, $84 \%, 81 \%$, and $95 \%$, respectively. The regression equations used to calculate the dietary concentrations from the intake of digestible nutrients were linear (Figure 1).
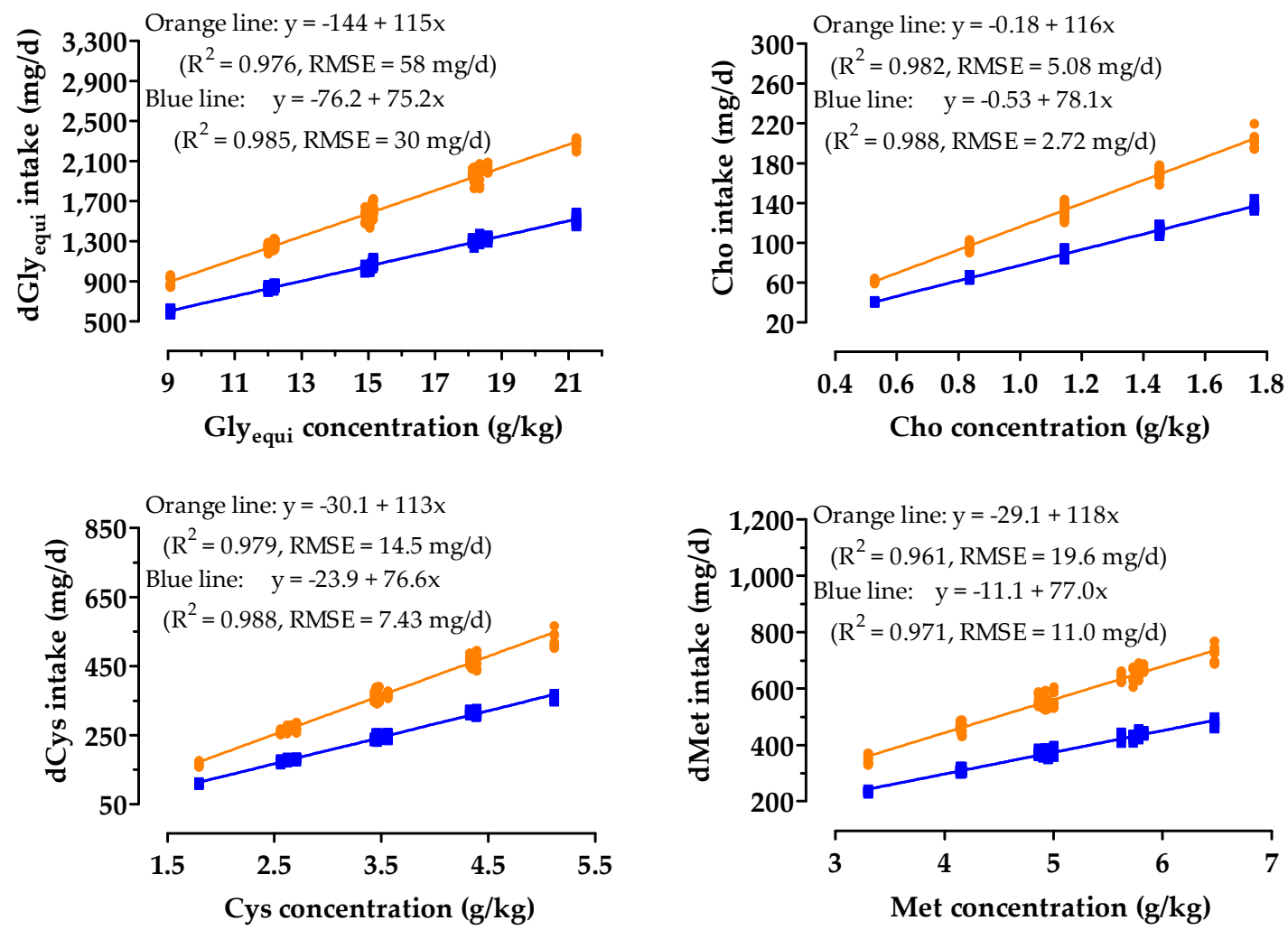

Figure 1. Relationship between diet concentrations and prececal digestible intake of glycine equivalent ( $\mathrm{dGly}_{\text {equi }}$ ), cysteine (dCys), methionine (dMet), and total choline (Cho) by broiler chickens from days 7 to 21 (blue lines) and days 18 to 21 (orange lines). y is the intake (mg/d) of dGlyequi, dCys or dMet or the total intake of Cho, and $\mathrm{x}$ is the dietary concentration $(\mathrm{g} / \mathrm{kg})$ of glycine equivalent (Gly equi), cysteine (Cys), methionine (Met), or Cho.

\subsection{Global Sensitivity Analysis}

Results of the global sensitivity analysis indicating the importance of independent variables (Table 6) were ranked via the intake of $\mathrm{dGly}_{\text {equi }}>\mathrm{dCys}>$ Cho for G:F, NUE, UA excretion, UA-N/(UA-N $+\mathrm{NH}_{3}-\mathrm{N}$ ) ratio, and plasma Gly concentration, the intake of $\mathrm{dCys}>\mathrm{Cho}>\mathrm{dGly}$ equi for $\mathrm{NH}_{3}$ excretion and plasma Met, Hcys, and Cysta concentrations, and the intake of $\mathrm{dGlyequi}>\mathrm{Cho}>\mathrm{dCys}$ for total $\mathrm{N}$ excretion. 
Table 3. Information and criteria of the fit of the neural network models for the prediction of gain:feed ratio of broiler chickens from days 7 to 21 , and excreta characteristics of broiler chickens from days 18 to 21 based on the dietary intake of digestible glycine equivalent, digestible cysteine, and total choline.

\begin{tabular}{|c|c|c|c|c|c|c|c|c|c|c|c|c|}
\hline \multirow[t]{2}{*}{ Item } & \multicolumn{2}{|c|}{ G:F } & \multicolumn{2}{|c|}{ NUE } & \multicolumn{2}{|c|}{ Daily $\mathrm{NH}_{3}$ Excretion } & \multicolumn{2}{|c|}{ Daily UA Excretion } & \multicolumn{2}{|c|}{$\begin{array}{l}\text { UA-N/(UA-N + } \\
\mathrm{NH}_{3} \text {-N) Ratio }\end{array}$} & \multicolumn{2}{|c|}{ Daily N Excretion } \\
\hline & $\begin{array}{c}\text { Training } \\
\text { Set }\end{array}$ & $\begin{array}{l}\text { Testing } \\
\text { Set }\end{array}$ & $\begin{array}{l}\text { Training } \\
\text { Set }\end{array}$ & $\begin{array}{c}\text { Testing } \\
\text { Set }\end{array}$ & $\begin{array}{c}\text { Training } \\
\text { Set }\end{array}$ & Testing Set & $\begin{array}{l}\text { Training } \\
\text { Set }\end{array}$ & Testing Set & $\begin{array}{c}\text { Training } \\
\text { Set }\end{array}$ & $\begin{array}{c}\text { Testing } \\
\text { Set }\end{array}$ & $\begin{array}{l}\text { Training } \\
\text { Set }\end{array}$ & $\begin{array}{l}\text { Testing } \\
\text { Set }\end{array}$ \\
\hline Type of network & \multicolumn{12}{|c|}{ Fully connected feed forward network } \\
\hline Training algorithm & \multirow{2}{*}{\multicolumn{12}{|c|}{$\begin{array}{l}\text { Evolutionary training using Genetic Algorithms } \\
\text { Genetic algorithms to identify neural network topology }\end{array}$}} \\
\hline Network structure selection & & & & & & & & & & & & \\
\hline $\begin{array}{c}\text { No. of observations in training and } \\
\text { testing set }\end{array}$ & 73 & 32 & 71 & 30 & 70 & 31 & 70 & 31 & 71 & 30 & 70 & 31 \\
\hline $\begin{array}{l}\text { Optimal number of hidden neurons } \\
\text { identified by genetic algorithms }\end{array}$ & \multicolumn{2}{|c|}{4} & \multicolumn{2}{|c|}{5} & \multicolumn{2}{|c|}{4} & \multicolumn{2}{|c|}{8} & \multicolumn{2}{|c|}{5} & \multicolumn{2}{|c|}{4} \\
\hline $\begin{array}{l}\text { Type of activation function in } \\
\text { hidden neuron }\end{array}$ & \multicolumn{2}{|c|}{ Hyperbolic tangent } & \multirow{2}{*}{\multicolumn{2}{|c|}{ Hyperbolic tangent }} & \multicolumn{2}{|c|}{ Hyperbolic tangent } & \multicolumn{2}{|c|}{ Hyperbolic tangent } & \multicolumn{2}{|c|}{ Hyperbolic tangent } & \multicolumn{2}{|c|}{ Hyperbolic tangent } \\
\hline Type of basing function in output neuron & \multicolumn{2}{|c|}{ Linear } & & & \multicolumn{2}{|c|}{ Linear } & \multicolumn{2}{|c|}{ Linear } & \multicolumn{2}{|c|}{ Linear } & \multicolumn{2}{|c|}{ Linear } \\
\hline$R^{2}$ & 0.75 & 0.90 & 0.60 & 0.66 & 0.83 & 0.90 & 0.83 & 0.84 & 0.85 & 0.82 & 0.54 & 0.63 \\
\hline $\begin{array}{c}\mathrm{R}^{2} \text { of the whole data set } \\
\text { Root MSE } \\
\text { Root MSE of the whole data set }\end{array}$ & $\begin{array}{r}0 \\
0.007 \mathrm{~g} / \mathrm{g} \\
0.0\end{array}$ & $\begin{array}{l}1 \\
\mathrm{~g} / \mathrm{g}\end{array}$ & $0.92 \%$ & $\%$ & $\begin{array}{r}0 \\
4.48 \mathrm{mg} / \mathrm{bird} \\
4.75\end{array}$ & $\begin{array}{l}86 \\
4.55 \mathrm{mg} / \mathrm{bird} \\
\mathrm{ng} / \mathrm{bird}\end{array}$ & $59.0 \mathrm{mg} / \mathrm{birc}$ & $\begin{array}{l}83 \\
54.0 \mathrm{mg} / \mathrm{bird} \\
\mathrm{ag} / \mathrm{bird}\end{array}$ & 0.01 & $\begin{array}{ll}4 \\
1\end{array}$ & $\begin{array}{r}0 \\
0.04 \mathrm{~g} / \mathrm{bird} \\
0.03\end{array}$ & $\begin{array}{l}56 \\
0.03 \mathrm{~g} / \mathrm{bird} \\
/ \mathrm{bird}\end{array}$ \\
\hline
\end{tabular}

G:F, gain:feed ratio; $\mathrm{NUE}$, nitrogen-utilization efficiency; $\mathrm{NH}_{3}$, ammonia; $\mathrm{UA}$, uric acid; UA-N/(UA-N + $\left.\mathrm{NH}_{3}-\mathrm{N}\right)$ ratio, uric acid-nitrogen/(uric acid-nitrogen + ammonia-nitrogen) ratio; $\mathrm{N}$, nitrogen.

Table 4. Information and criteria of the fit of the neural network models for the prediction of some plasma amino acids and biogenic amines in broiler chickens on day 22 based on a dietary intake of digestible glycine equivalent, digestible cysteine, and total choline.

\begin{tabular}{|c|c|c|c|c|c|c|c|c|}
\hline \multirow[b]{2}{*}{ Item } & \multicolumn{2}{|c|}{ Gly } & \multicolumn{2}{|c|}{ Met } & \multicolumn{2}{|c|}{ Hcys ${ }^{1}$} & \multicolumn{2}{|c|}{ Cysta $^{2}$} \\
\hline & Training Set & Testing Set & Training Set & Testing Set & Training Set & Testing Set & Training Set & Testing Se \\
\hline $\mathrm{n}$ & \multicolumn{8}{|c|}{ Fully connected feed forward network } \\
\hline Training algorithm & \multirow{2}{*}{\multicolumn{8}{|c|}{$\begin{array}{l}\text { Evolutionary training using Genetic Algorithms } \\
\text { Genetic algorithms to identify neural network topology }\end{array}$}} \\
\hline Network structure selection & & & & & & & & \\
\hline No. of observations in training and testing set & 72 & 31 & 72 & 31 & 72 & 31 & 64 & 28 \\
\hline $\begin{array}{l}\text { Optimal number of hidden neurons identified } \\
\text { by genetic algorithms }\end{array}$ & \multicolumn{2}{|c|}{7} & \multicolumn{2}{|c|}{7} & \multicolumn{2}{|c|}{7} & \multicolumn{2}{|c|}{6} \\
\hline Type of activation function in hidden neuron & \multirow{2}{*}{\multicolumn{2}{|c|}{$\begin{array}{l}\text { Hyperbolic tangent } \\
\text { Linear }\end{array}$}} & \multirow{2}{*}{\multicolumn{2}{|c|}{$\begin{array}{l}\text { Hyperbolic tangent } \\
\text { Linear }\end{array}$}} & \multirow{2}{*}{\multicolumn{2}{|c|}{$\begin{array}{l}\text { Hyperbolic tangent } \\
\text { Linear }\end{array}$}} & \multirow{2}{*}{\multicolumn{2}{|c|}{$\begin{array}{l}\text { Hyperbolic tangent } \\
\text { Linear }\end{array}$}} \\
\hline Type of basing function in output neuron & & & & & & & & \\
\hline $\mathrm{R}^{2}$ & 0.81 & 0.77 & 0.67 & 0.67 & 0.50 & 0.65 & 0.47 & 0.63 \\
\hline$R^{2}$ of the whole data set & \multicolumn{2}{|c|}{0.80} & \multicolumn{2}{|c|}{0.67} & \multicolumn{2}{|c|}{0.55} & \multirow{2}{*}{\multicolumn{2}{|c|}{0.51}} \\
\hline Root MSE (mg/100 mL) & 1.45 & 1.69 & 0.27 & 0.24 & 0.05 & 0.07 & & \\
\hline Root MSE of the whole data set $(\mathrm{mg} / 100 \mathrm{~mL})$ & \multicolumn{2}{|c|}{1.53} & \multicolumn{2}{|c|}{0.26} & \multicolumn{2}{|c|}{0.06} & \multicolumn{2}{|c|}{0.09} \\
\hline
\end{tabular}

${ }^{1} 55$ observations set to 0 because no concentrations were detectable, and the lowest quantified concentration of $0.05 \mathrm{mg} / 100 \mathrm{~mL}$ was close to 0 ; ${ }^{2} 11$ observations were not used for data evaluation because no concentrations were quantified, and the lowest detected concentration $(0.16 \mathrm{mg} / 100 \mathrm{~mL})$ was not close to 0; Gly, glycine; Met, methionine; Hcys, homocystine; Cysta, cystathionine. 
Table 5. Prececal digestibility (\%, arithmetic mean \pm SD) of crude protein and amino acids in the digestibility trial diet $(n=5)$.

\begin{tabular}{cc}
\hline Item & Prececal Digestibility \\
\hline Crude protein & $91 \pm 0.8$ \\
Lysine & $95 \pm 0.9$ \\
Methionine & $95 \pm 0.9$ \\
Cysteine & $81 \pm 1.2$ \\
Threonine & $89 \pm 0.9$ \\
Tryptophan & $90 \pm 1.8$ \\
Arginine & $96 \pm 0.6$ \\
Valine & $93 \pm 0.6$ \\
Isoleucine & $92 \pm 0.8$ \\
Leucine & $94 \pm 0.6$ \\
Histidine & $91 \pm 0.8$ \\
Phenylalanine & $96 \pm 0.7$ \\
Glycine & $84 \pm 1.5$ \\
Serine & $84 \pm 1.2$ \\
Alanine & $93 \pm 0.8$ \\
Aspartic acid/asparagine & $93 \pm 0.8$ \\
Glutamic acid/glutamine & $92 \pm 0.7$ \\
Proline & $92 \pm 0.4$ \\
\hline
\end{tabular}

Table 6. Variable total effect in the neural network models for the prediction of gain:feed ratio, excreta characteristics, and plasma metabolites.

\begin{tabular}{|c|c|c|c|}
\hline Item & dGly equi Intake & dCys Intake & Cho Intake \\
\hline \multicolumn{4}{|l|}{ G:F } \\
\hline Sensitivity & 0.99 & 0.04 & 0.02 \\
\hline Rank & 1 & 2 & 3 \\
\hline \multicolumn{4}{|l|}{ NUE } \\
\hline Sensitivity & 0.78 & 0.55 & 0.35 \\
\hline Rank & 1 & 2 & 3 \\
\hline \multicolumn{4}{|c|}{ Total $\mathrm{N}$ excretion } \\
\hline Sensitivity & 0.93 & 0.08 & 0.09 \\
\hline Rank & 1 & 3 & 2 \\
\hline \multicolumn{4}{|l|}{ UA excretion } \\
\hline Sensitivity & 0.89 & 0.19 & 0.11 \\
\hline Rank & 1 & 2 & 3 \\
\hline \multicolumn{4}{|l|}{$\mathrm{NH}_{3}$ excretion } \\
\hline Sensitivity & 0.32 & 0.72 & 0.38 \\
\hline Rank & 3 & 1 & 2 \\
\hline \multicolumn{4}{|c|}{ UA-N/(UA-N + $\left.\mathrm{NH}_{3}-\mathrm{N}\right)$ ratio } \\
\hline Sensitivity & 0.63 & 0.54 & 0.06 \\
\hline Rank & 1 & 2 & 3 \\
\hline \multicolumn{4}{|c|}{ Plasma Gly concentration } \\
\hline Sensitivity & 0.97 & 0.06 & 0.04 \\
\hline Rank & 1 & 2 & 3 \\
\hline \multicolumn{4}{|c|}{ Plasma Met concentration } \\
\hline Sensitivity & 0.05 & 0.81 & 0.20 \\
\hline Rank & 3 & 1 & 2 \\
\hline \multicolumn{4}{|c|}{ Plasma Hcys concentration } \\
\hline Sensitivity & 0.15 & 0.70 & 0.20 \\
\hline Rank & 3 & 1 & 2 \\
\hline \multicolumn{4}{|c|}{ Plasma Cysta concentration } \\
\hline Sensitivity & 0.10 & 0.87 & 0.13 \\
\hline Rank & 3 & 1 & 2 \\
\hline
\end{tabular}

dGlyequi, digestible glycine equivalent; dCys, digestible cysteine; Cho, choline; G:F, gain:feed ratio; $\mathrm{NUE}$, nitrogen-utilization efficiency; $\mathrm{N}$, nitrogen; $\mathrm{UA}$, uric acid; $\mathrm{NH}_{3}$, ammonia; $\mathrm{UA}-\mathrm{N} /\left(\mathrm{UA}-\mathrm{N}+\mathrm{NH}_{3}-\mathrm{N}\right)$ ratio, uric acid-nitrogen/(uric acid-nitrogen + ammonia-nitrogen) ratio; Gly, glycine; Met, methionine; Hcy, homocystine; Cysta, cystathionine. 


\subsection{Average Daily Gain and Average Daily Feed Intake}

Neural networks showed strong relationships among the nutrients and ADG and ADFI $\left(R^{2} \geq 0.93\right)$, and variation in these traits was low (57.0-68.7 g/bird for ADG and 72.7-82.9 g/bird for ADFI). However, the modeled curve shapes of these traits were undulated (Figures S1 and S2) and, therefore, biologically implausible. Similar high $\mathrm{R}^{2}$ and undulated curve shapes were obtained when neural networks with a high number of preset model parameter combinations were used instead of using combinations of model parameters that were obtained from genetic algorithms. Furthermore, data analysis by using full quadratic models including interaction effects did not adequately describe ADG and ADFI (data not shown). We concluded that the obtained models for ADG and ADFI from all evaluations were unsuitable, and these traits are not further described herein.

\subsection{Gain:Feed Ratio}

Values of G:F from days 7 to 21 increased with dGly equi $_{\text {intake, particularly up to a dGly }}$ equi intake of $900 \mathrm{mg} /$ day (Figure 2). Overall, G:F increased with higher dCys intake. The highest difference in G:F between high and low dCys intake was $0.011 \mathrm{~g} / \mathrm{g}$ at a dGlyequi intake of $956 \mathrm{mg} /$ day. The G:F was approximately $0.83 \mathrm{~g} / \mathrm{g}$ with different dCys intakes and at a dGly equi intake above $1170 \mathrm{mg} /$ day. An effect of Cho intake on G:F was barely pronounced compared with that of dGly $y_{\text {equi }}$ and dCys intake. G:F slightly increased with low Cho and high dCys intake and the highest difference between low and high Cho intake was $0.004 \mathrm{~g} / \mathrm{g}$ at $1034 \mathrm{mg}$ of dGlyequi intake/day.
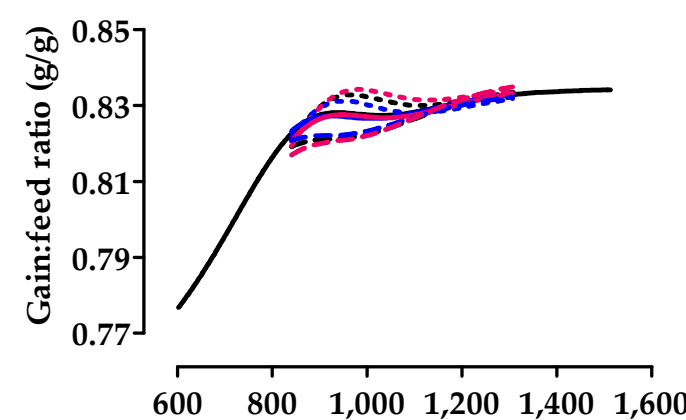

$[9.0][11.6][14.3][17.0][19.6][22.3]$

dGly $_{\text {equi }}$ intake (mg/d)

[Gly equi $\left._{\text {concentration }}(\mathrm{g} / \mathrm{kg})\right]$

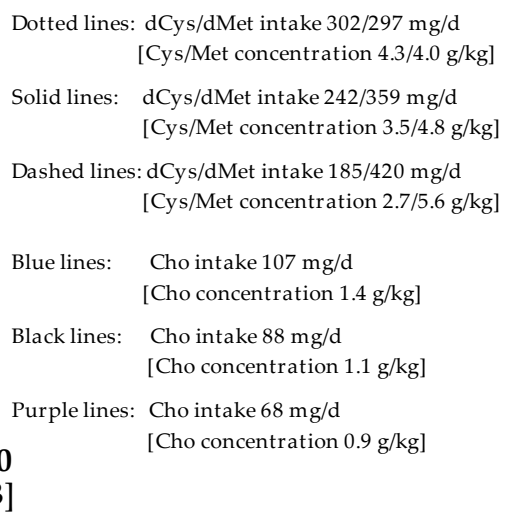
]

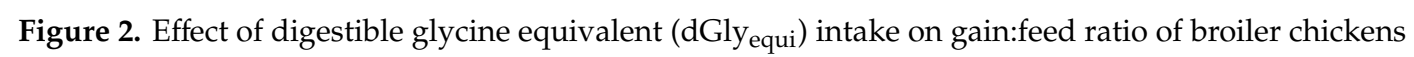
at varying digestible cysteine ( $\mathrm{dCys}$ ) and choline (Cho) intake from days 7 to 21 . $\mathrm{dCys}$ and Cho intake levels correspond to level $-1,0$, and +1 based on the fractional central composite design.

\subsection{Nitrogen-Utilization Efficiency}

Values of NUE varied between $76.7 \%$ and $80.2 \%$ for medium Cho intake (Figure 3a). The NUE

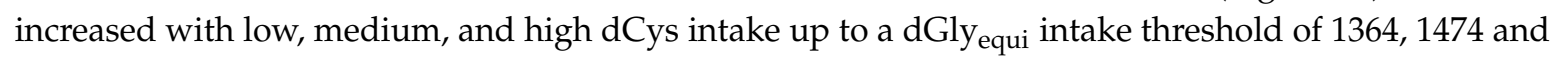
$1392 \mathrm{mg} /$ day, respectively, and decreased at higher intake levels of dGlyequi. The NUE was the highest at medium dCys intake; it was higher at low dCys intake than at high dCys intake. Differences in NUE with different Cho intakes and with low dCys intake were small (Figure 3b). At medium dCys intake (Figure 3c), the influence of high and low Cho intake on NUE up to a dGlyequi intake of approximately $1650 \mathrm{mg} / \mathrm{d}$ was low. At high Glyequi intake, NUE increased and decreased with low and high Cho intake, respectively. With high dCys intake (Figure 3d), the response to dGly equi was similar with different Cho intake levels, with higher NUE at the low Cho intake compared to medium and high Cho intake. 




Dotted lines: dCys/dMet intake 436/466 mg/d [Cys/Met concentration 4.1/4.2 g/kg]

Solid lines: dCys/dMet intake 364/537 mg/d [Cys/Met concentration 3.5/4.8 g/kg]

Dashed lines: dCys/dMet intake 287/620 mg/d [Cys/Met concentration 2.8/5.5 g/kg]

$\begin{array}{cl}\text { Blue lines: } & \begin{array}{l}\text { Cho intake } 158 \mathrm{mg} / \mathrm{d} \\ \text { [Cho concentration } 1.4 \mathrm{~g} / \mathrm{kg} \text { ] }\end{array} \\ \text { Black lines: } & \begin{array}{l}\text { Cho intake } 132 \mathrm{mg} / \mathrm{d} \\ \text { [Cho concentration } 1.1 \mathrm{~g} / \mathrm{kg} \text { ] }\end{array} \\ \text { Purple lines: } & \text { Cho intake } 103 \mathrm{mg} / \mathrm{d} \\ & {[\text { Cho concentration } 0.9 \mathrm{~g} / \mathrm{kg} \text { ] }}\end{array}$
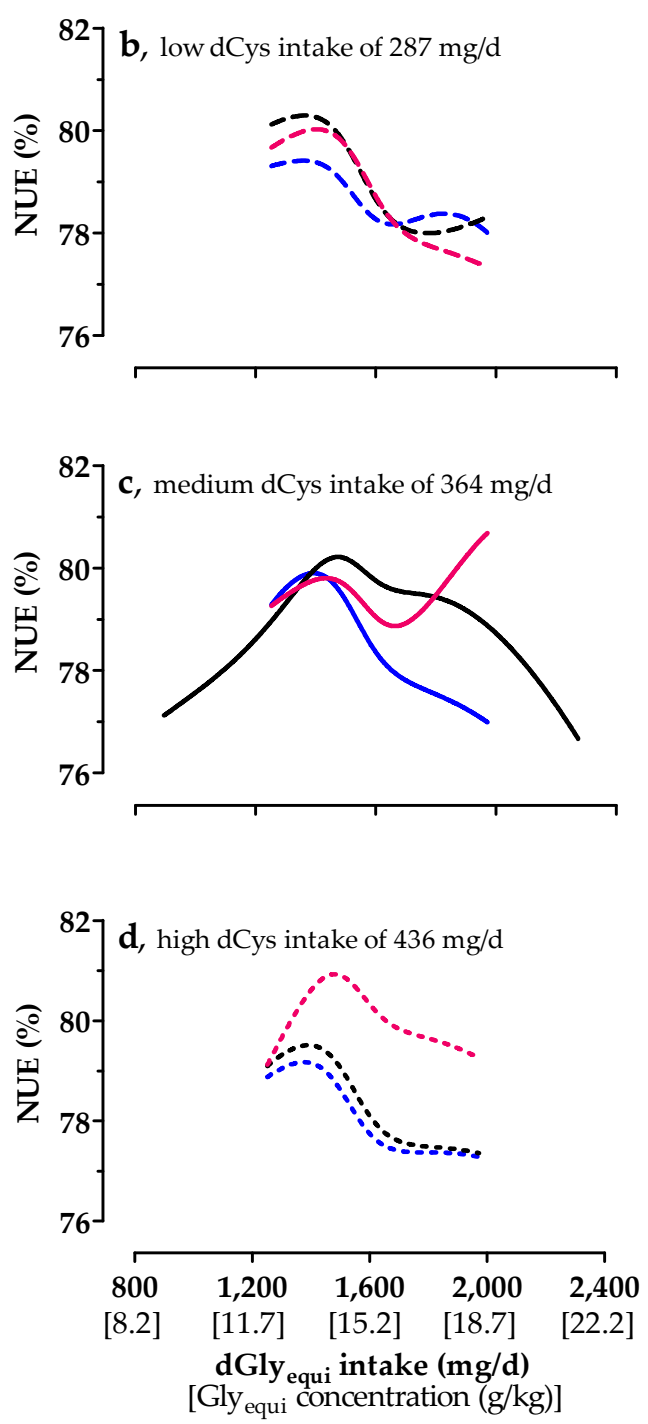

Figure 3. Effect of digestible glycine equivalent ( $\left.\mathrm{dGly}_{\text {equi }}\right)$ intake on nitrogen-utilization efficiency (NUE) of broiler chickens at varying digestible cysteine (dCys) and medium choline (Cho) intake from days 18 to 21 (a), and the effect of dGly equi intake on the NUE of broiler chickens at varying Cho and low, medium, and high dCys intake from days 18 to 21 (b-d). dCys and Cho intake levels correspond to level $-1,0$, and +1 based on the fractional central composite design.

\subsection{Nitrogen Excretion}

Total $\mathrm{N}$ and UA excretion increased with medium Cho intake when dGlyequi intake was higher than 1250 and $1410 \mathrm{mg} /$ day, respectively (Figures 4a and 5a). Lower dGlyequi intake did not influence total $\mathrm{N}$ and UA excretion. $\mathrm{NH}_{3}$ excretion decreased up to a dGlyequi intake of approximately $1700 \mathrm{mg} / \mathrm{day}$ and slightly increased at higher intake levels (Figure 5e). $\mathrm{NH}_{3}$ and UA excretion were the highest with high dCys intake. The intake of dCys hardly influenced total $\mathrm{N}$ excretion. Almost no differences in total $\mathrm{N}$ and UA excretion were found among different Cho intakes and with low dCys intake (Figures $4 \mathrm{~b}$ and $5 \mathrm{~b}$ ). With medium and high $\mathrm{dCys}$ intake, total $\mathrm{N}$ and UA excretion were the lowest with low Cho intake (Figure $4 \mathrm{c}$, d and Figure $5 \mathrm{c}, \mathrm{d}$ ). $\mathrm{NH}_{3}$ excretion was almost unaffected by low and medium Cho intake at low and medium dCys intake (Figure 5f,g). At high dCys intake, $\mathrm{NH}_{3}$ excretion with medium Cho intake was the highest up to a dGlyequi intake of $1545 \mathrm{mg} /$ day and decreased with increasing dGly equi intake to the level that was excreted with low Cho intake (Figure 5h). Increasing




The UA-N/(UA-N $\left.+\mathrm{NH}_{3}-\mathrm{N}\right)$ ratio was almost unaffected by Cho intake and was slightly higher with low Cho intake.

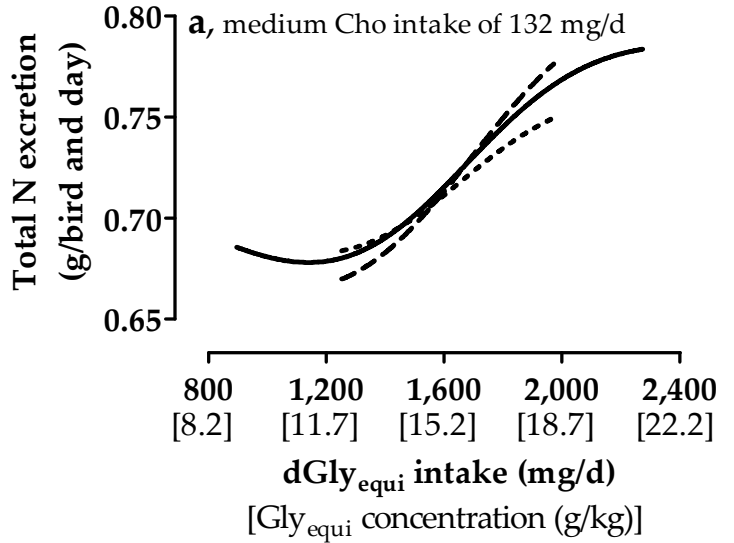

Dotted lines: dCys/dMet intake 436/466 mg/d [Cys/Met concentration 4.1/4.2 g/kg]

Solid lines: dCys/dMet intake $364 / 537 \mathrm{mg} / \mathrm{d}$ [Cys/Met concentration 3.5/4.8 g/kg]

Dashed lines: dCys/dMet intake 287/620 mg/d

[Cys/Met concentration 2.8/5.5 g/kg]

Blue lines: Cho intake $158 \mathrm{mg} / \mathrm{d}$

[Cho concentration $1.4 \mathrm{~g} / \mathrm{kg}$ ]

Black lines: Cho intake $132 \mathrm{mg} / \mathrm{d}$

[Cho concentration $1.1 \mathrm{~g} / \mathrm{kg}$ ]

Purple lines: Cho intake $103 \mathrm{mg} / \mathrm{d}$

[Cho concentration $0.9 \mathrm{~g} / \mathrm{kg}$ ]
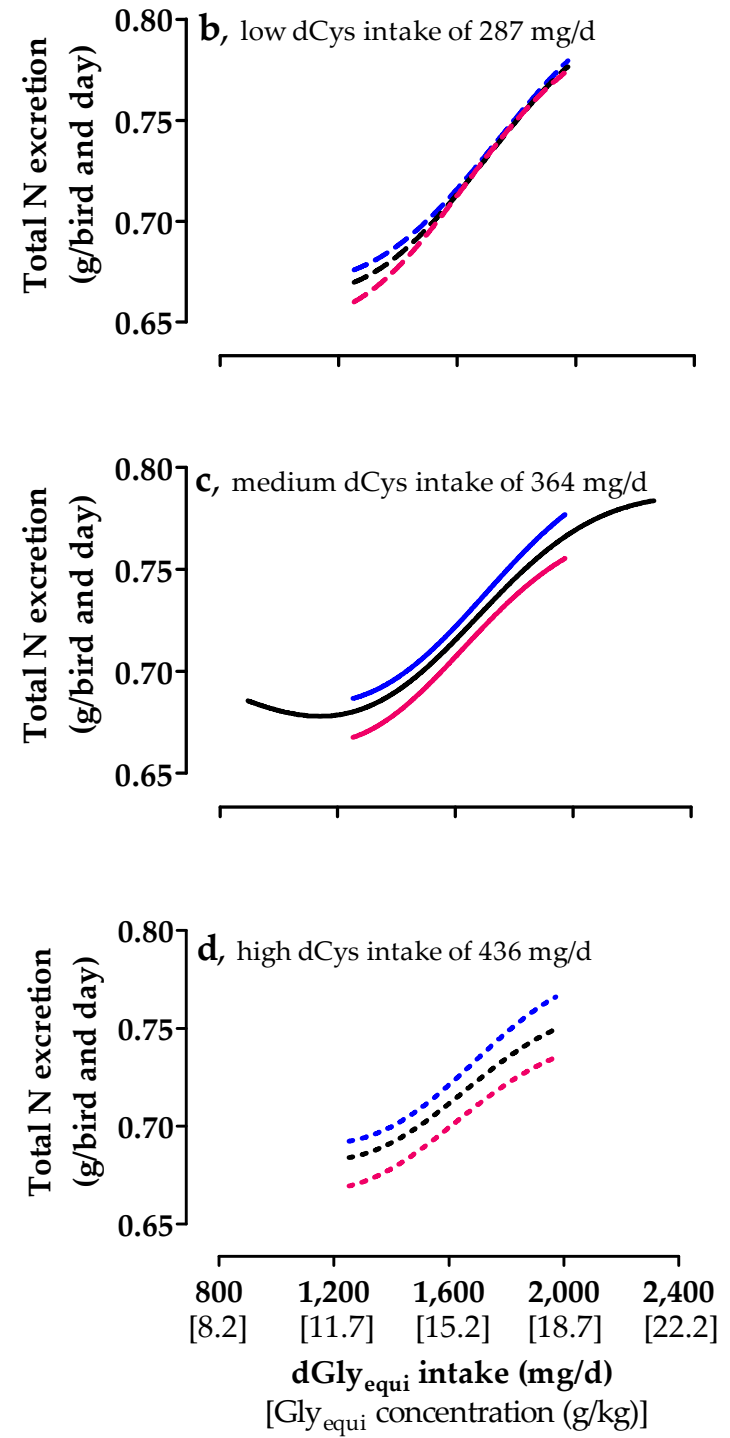

Figure 4. Effect of digestible glycine equivalent (dGly equi) intake on total nitrogen $(\mathrm{N})$ excretion of broiler chickens at varying digestible cysteine (dCys) and medium choline (Cho) intake from days 18 to 21 (a), and effect of dGly equi intake on total $\mathrm{N}$ excretion of broiler chickens at varying Cho and low, medium, and high dCys intake from days 18 to $21(\mathbf{b}-\mathbf{d})$. dCys and Cho intake levels correspond to level $-1,0$, and +1 based on the fractional central composite design. 
Dotted lines: dCys/dMet intake 436/466 mg/d [Cys/Met concentration 4.1/4.2 g/kg]

Solid lines: dCys/dMet intake 364/537 mg/d [Cys/Met concentration 3.5/4.8 g/kg]

Dashed lines: dCys/dMet intake 287/620 mg/d [Cys/Met concentration 2.8/5.5 g/kg]
Blue lines:

Cho intake $158 \mathrm{mg} / \mathrm{d}$

[Cho concentration $1.4 \mathrm{~g} / \mathrm{kg}$ ]

Black lines: Cho intake $132 \mathrm{mg} / \mathrm{d}$

[Cho concentration $1.1 \mathrm{~g} / \mathrm{kg}$ ]

Purple lines: Cho intake $103 \mathrm{mg} / \mathrm{d}$

[Cho concentration $0.9 \mathrm{~g} / \mathrm{kg}$ ]
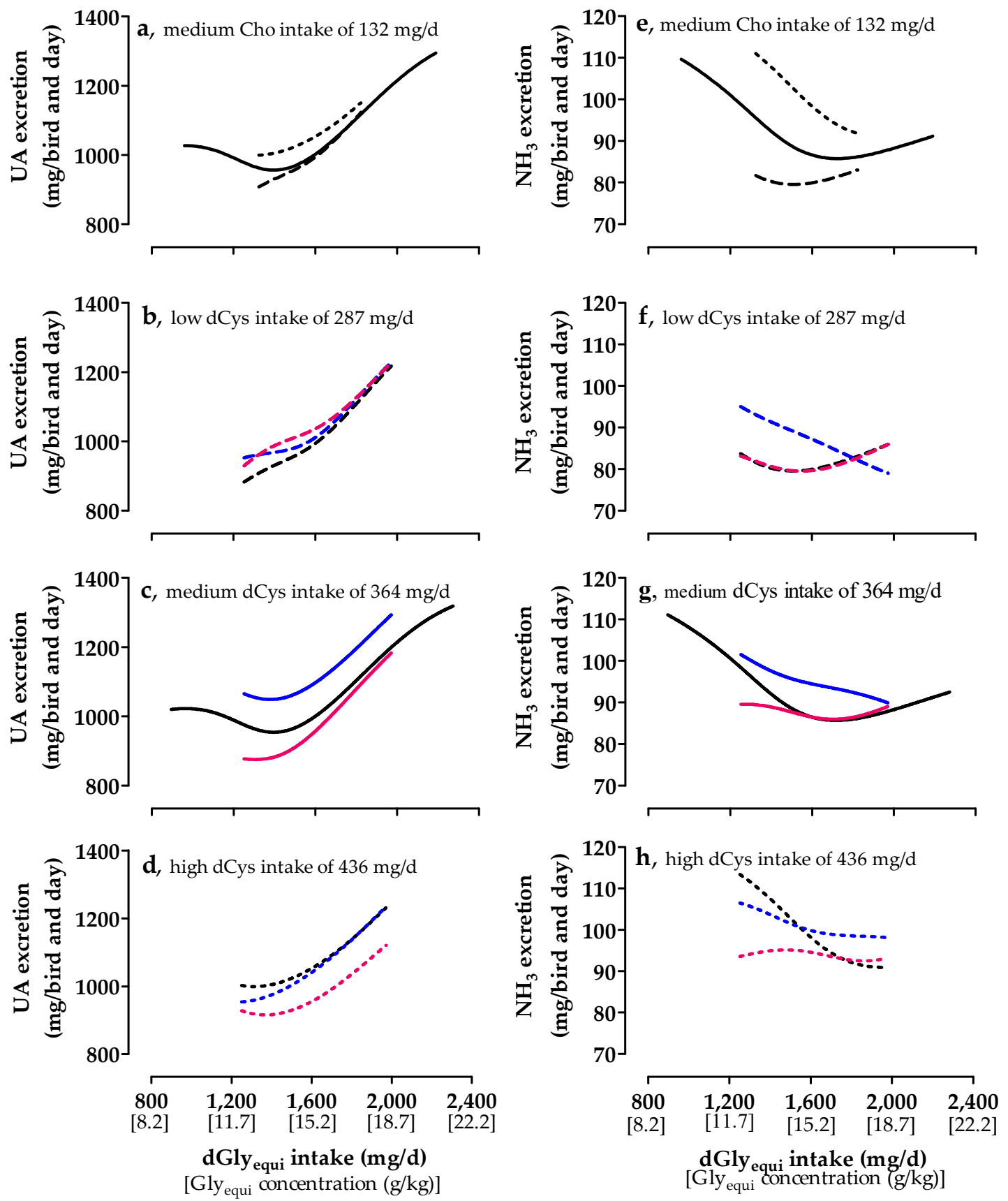

Figure 5. Effect of digestible glycine equivalent (dGlyequi) intake on uric acid (UA) (a) and ammonia $\left(\mathrm{NH}_{3}\right)(\mathbf{e})$ excretion of broiler chickens at varying digestible cysteine (dCys) and medium choline (Cho) intake from days 18 to 21, and the effect of dGly equi intake on UA (b-d) and $\mathrm{NH}_{3}(\mathbf{f}-\mathbf{h})$ excretion of broiler chickens at varying Cho and low, medium, and high dCys intake from days 18 and 21. dCys and Cho intake levels correspond to level $-1,0$, and +1 based on the fractional central composite design. 




Figure 6. Effect of digestible glycine equivalent (dGlyequi) intake on uric acid-nitrogen/(uric acid-nitrogen + ammonia-nitrogen) ratio ((UA-N/(UA-N $\left.+\mathrm{NH}_{3}-\mathrm{N}\right)$ ratio) in the excreta of broiler chickens at varying digestible cysteine (dCys) and choline (Cho) intake from days 18 to 21 . dCys and Cho intake levels correspond to level $-1,0$, and +1 based on the fractional central composite design.

\subsection{Plasma Metabolites}

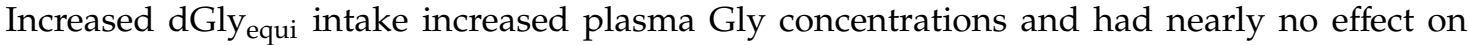
plasma Met concentrations (Figure 7a,b). Plasma Hcys concentrations decreased and plasma Cysta concentrations increased at low and medium dCys intake when dGly equi intake increased (Figure 7c,d). Concentrations of these compounds were almost unaffected at high dCys intake. Plasma Met, Hcys, and Cysta concentrations decreased with increasing dCys intake. Plasma Gly concentrations were almost unaffected by dCys intake. Cho intake had nearly no effect on plasma Gly concentrations (Figure 7a). Higher Cho intake increased plasma Met concentrations irrespective of dCys intake (Figure $7 \mathrm{~b}$ ) and decreased plasma Hcys concentrations at low and medium dCys intake (Figure 7c). Plasma Hcys concentrations were almost unaffected by Cho intake at high dCys intake. The lowest Cho intake resulted in the highest plasma Cysta concentrations, and the difference in this concentration was hardly pronounced between medium and high Cho intake (Figure 7d). 




Dotted lines: dCys/dMet intake 302/297 mg/d [Cys/Met concentration $4.3 / 4.0 \mathrm{~g} / \mathrm{kg}$ ]

Solid lines: dCys/dMet intake 242/359 mg/d [Cys/Met concentration 3.5/4.8 g/kg]

Dashed lines: dCys/dMet intake 185/420 mg/d [Cys/Met concentration 2.7/5.6 g/kg]

Blue lines:

Cho intake $107 \mathrm{mg} / \mathrm{d}$

[Cho concentration $1.4 \mathrm{~g} / \mathrm{kg}$ ]

Black lines: Cho intake $88 \mathrm{mg} / \mathrm{d}$ [Cho concentration $1.1 \mathrm{~g} / \mathrm{kg}$ ]

Purple lines: Cho intake $68 \mathrm{mg} / \mathrm{d}$ [Cho concentration $0.9 \mathrm{~g} / \mathrm{kg}$ ]


Figure 7. Effect of digestible glycine equivalent (dGly equi) intake with varying digestible cysteine (dCys) and choline (Cho) intake from days 7 to 21 on plasma glycine (Gly) (a), methionine (Met) (b), homocystine (Hcys) (c), and cystathionine (Cysta) (d) concentrations of broiler chickens on day 22. dCys and Cho intake levels correspond to level $-1,0$, and +1 based on the fractional central composite design.

\section{Discussion}

The objective of the present study was to investigate the interactive effects among dietary Gly equi, Cys, and Cho on growth. The interactive effects among these compounds was noted on G:F; however, they were small, although the metabolism of these compounds was influenced, as shown from the plasma metabolite concentrations. More pronounced interactive effects between Gly equi and Cys [6] and

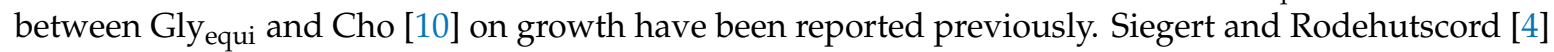
suggested that the extent of the interactive effects between Glyequi and Cys, or Gly equi and Cho on growth may be affected by varying NUE and urinary N excretion because the formation of UA requires Gly. The outcomes of the present study support this suggestion.

\subsection{Effects of Glyequi}

G:F was predominately influenced by the $\mathrm{dGly}_{\text {equi }}$ intake as indicated by the global sensitivity analysis. The increased NUE up to a dGly equi intake of approximately $1470 \mathrm{mg} /$ day with medium $\mathrm{dCys}$ and Cho intake showed that the more of the ingested $\mathrm{N}$ was accreted the less limiting Glyequi intake was. This likely led to a higher G:F. NUE decreased with dGlyequi intake above $1470 \mathrm{mg} /$ day 
because the additional $\mathrm{Gly}_{\text {equi }}$ could not be used by the animals, and the $\mathrm{N}$ contained in the surplus $\mathrm{Gly}_{\text {equi }}$ had to be excreted. Domestic fowl predominately excretes $\mathrm{N}$ in the urine as UA, with urinary $\mathrm{N}$ consisting of $72 \%-91 \%$ of $\mathrm{UA}-\mathrm{N}$ plus $\mathrm{NH}_{3}-\mathrm{N}$ [23]. One molecule of Gly is required for the formation of one molecule of UA [11]. A surplus dGly equi intake above $1470 \mathrm{mg} /$ day was mostly excreted as UA because UA excretion and the UA-N/(UA-N $\left.+\mathrm{NH}_{3}-\mathrm{N}\right)$ ratio increased with increasing dGly equi intake. Therefore, dietary Gly equi fed in excess was disadvantageous when the aim was to increase NUE.

\subsection{Consequences of High Nitrogen-Utilization Efficiency}

Varying NUE is a considerable factor that influences the effects of dietary Gly equi, Cys, and Cho. The observed NUE of $77 \%$ to $80 \%$ was very high compared to that found in the literature, where NUE ranged between $50 \%$ and $65 \%$ for diets containing 240 and $170 \mathrm{~g} \mathrm{CP} / \mathrm{kg}$ [24-26]. A high NUE indicates low catabolism of AA, meaning that digestible AA concentrations in the diets were close to the requirement of the animals. The Gly equi requirement of broiler chickens decreases when NUE increases because a lower proportion of ingested $\mathrm{N}$ has to be excreted via the urine as UA, for which Gly is dissipated [4]. Thus, the high NUE found in the present study contributed to a low Gly equi requirement of the birds. A value of $95 \%$ of maximum response, often defined as the recommended concentration of a nutrient [10,27], at a medium dCys and Cho intake was obtained at a dGlyequi intake of $684 \mathrm{mg} /$ day (equivalent to $10 \mathrm{~g} \mathrm{Gly}$ equi $/ \mathrm{kg}$ ). This is at the lower range of the dietary Glyequi values required for broiler chickens during the first 3 weeks of age to achieve $95 \%$ of the maximum G:F that has been reported to vary between 10 and $16 \mathrm{~g}$ Gly equi $/ \mathrm{kg}$ in previous studies [4]. Additional Glyequi was possibly available when dCys (because of less Ser required to form Cys from Met) and Cho (because it is an endogenous precursor of Gly) intake increased. However, the additional Gly equi obtained by these processes could not be used by the animals for G:F because the birds were abundantly supplied with Gly equi in the investigated range of dGly equi where the interactions with $\mathrm{dCys}$ and Cho could be determined (levels -1 to +1 based on the study design corresponding to 840 and $1310 \mathrm{mg}$ dGly equi intake/day, respectively). This could explain the small effects of varying dCys and Cho intake on G:F found in the present study. More pronounced interactive effects of dGlyequi, $\mathrm{dCys}$, and Cho intake on G:F would likely have occurred at a dGlyequi intake below $840 \mathrm{mg} / \mathrm{d}$. The variation in NUE was small, which contributed to a similar Gly equi requirement for UA excretion among treatments. Taken together, the high level and low variation in NUE resulted in a low Gly equi requirement, which explains the small pronounced effects of dietary Cys and Cho on the response to dietary Glyequi on growth.

This contradicts the findings by Siegert et al. [6], who found that the growth response to

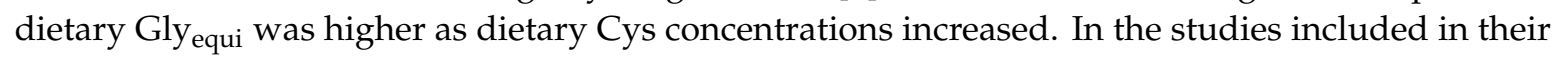
meta-analysis, dietary Cys was positively correlated with dietary $\mathrm{CP}$, which varied between 160 and $240 \mathrm{~g} / \mathrm{kg}$. NUE was not determined in the studies included in their meta-analysis; however, the varying dietary $\mathrm{CP}$ most likely influenced NUE. In contrast to the meta-analysis by Siegert et al. [6], the variation of dietary $\mathrm{CP}$ and NUE in the present study was low and $\mathrm{CP}$ and Cys concentrations in the diets were not correlated $(P=0.691)$. Therefore, the effects of different Cys concentrations in the present study were almost unaffected by the effects of different dietary CP on NUE and resulting implications on interactive effects of Glyequi and Cys on G:F. The Cys effect found in the previous meta-analysis could have been an artifact caused by a concurrently variable $\mathrm{CP}$ supply.

\subsection{Effects of Cysteine Formation from Methionine}

Different dCys intake influenced Met conversion to Cys. This probably contributed to the overall highest G:F at high dCys intake and to higher G:F response with dGlyequi intake at low dCys intake compared to that at high dCys intake. These findings are consistent with the results of Siegert et al. [6] and Powell et al. [28]. The formation of Cys from Met produces Hcy and Cysta as intermediates [5]. The plasma concentrations of Hcys and Cysta varied depending on dGlyequi and dCys intake in the present study. The decreasing plasma Hcys and Cysta concentrations indicated that lower proportions of Met were converted to Cys with higher dCys intake. One molecule of Ser is required when Hcy 
is converted to Cysta [5,29], and one molecule of ammonia is produced when Cysta is metabolized to Cys [29]. Ammonia can be converted to UA during the dissipation of Gly equi or it can be used to produce nonessential AA [4,30,31]. Therefore, every molecule of Met that is not metabolized to Cys spares one or two Glyequi molecules. Thus, Glyequi is more available for other metabolic processes such as $\mathrm{N}$ accretion or UA formation and could explain the slightly higher G:F and UA excretion at higher dCys intake. Conversely, more Glyequi was required for Cys formation from Met to meet bird Cys requirement at lower dCys intake, which would explain the higher G:F response to Glyequi supplementation at lower dCys intake than that at higher dCys intake.

\subsection{Effects of Choline}

A low conversion of Cho to Gly might be an explanation for the smaller extent of interactive effects of Glyequi and Cho compared to those of Glyequi and Cys on G:F. Increasing plasma concentrations of Met together with decreasing plasma concentrations of Hcys and Cysta with increasing Cho intake indicated that a higher proportion of Hcy was remethylated to Met. Remethylation of Hcy to Met can occur via the BHMT and MS pathways [29]. Via BHMT, Cho is metabolized to betaine and then to dimethylglycine when one methyl group of betaine is delivered to Hcy. Subsequently, dimethylglycine is converted to Gly. Results of studies describing whether BHMT or MS prevail upon Cho supplementation are not uniform. It was reported that Cho supplementation decreased the proportion of Hcy remethylated via BHMT [32,33] and downregulated BHMT activity [34] in broiler chickens. In this case, Cho would deliver methyl groups for the remethylation of Hcy via MS [32,33] without Gly being produced. In other studies, Cho supplementation increased BHMT activity in broiler chickens [35], pigs [36], and rats [37]. A higher activity of BHMT led to an increased formation of Gly. The reasons for these inconsistent effects of Cho supplementation on BHMT and MS activities are unclear. Possibly, the effects of Cho supplementation on BHMT and MS activities diverged among studies and, therefore, contributed to a different extent of interactive effects between dietary Cho and Gly $_{\text {equi }}$ on growth between the results by Siegert et al. [10] and the present study.

\subsection{Effect of Digestible Cysteine Intake on Acid Excretion}

Higher dCys intake led to higher acid excretion. Increased acid excretion was indicated by the $\mathrm{UA}-\mathrm{N} /\left(\mathrm{UA}-\mathrm{N}+\mathrm{NH}_{3}-\mathrm{N}\right)$ ratio that increased with increasing dCys intake. The increasing UA-N/(UA-N + $\mathrm{NH}_{3}-\mathrm{N}$ ) ratio was not caused by Gly equi deficiency limiting UA formation as described previously $[14,19]$ because Gly equi was adequately supplied. Dietary Cys increased by adding Cys as free L-Cys $\cdot \mathrm{HCl} \cdot \mathrm{H}_{2} \mathrm{O}$, inducing an acid load in the birds owing to the contained hydrochloride [38]. Therefore, it was more likely that higher $\mathrm{NH}_{3}$ excretion was an adaption to the excretion of acid loads to maintain the acid-base balance [39]. Consequences of an altered acid-base balance have the potential to decrease the growth of broiler chickens [40,41]. For the present study, however, the mechanisms of the animals to maintain an acid-base balance were not challenged to an extent that decreased their growth because high dCys intake led to an overall high G:F.

\subsection{Neural Network and Genetic Algorithm}

Neural networks can be utilized to model, analyze, and optimize the response of broiler chickens to dietary nutrient concentrations or intake [10,42]. The optimal number of hidden neurons and their respective coefficients must be determined for maximized prediction accuracy. In previous studies, neural networks were often designed using a time-consuming iterative trial and error approach that requires much experience in developing neural networks by the operator. Using genetic algorithms and neural networks together can overcome these disadvantage [43-45]. The results of the present study indicate that using genetic algorithms for training neural networks decreases operational effort, making the architecture of neural networks less dependent of the operator. This might improve the applicability of neural networks in animal nutrition science. 


\section{Conclusions}

The interactive effects of dietary Gly equi, Cys, and Cho on performance were slightly pronounced, possibly because NUE was high and barely influenced by the variation of these nutrients. The interactive effects between Glyequi and Cho on growth were smaller than those between Glyequi and Cys. Plasma metabolites indicated that the metabolism of Glyequi, Cys, and Cho was influenced by varying concentrations of these nutrients; however, the consequences for growth were small.

Supplementary Materials: The following are available online at http:/www.mdpi.com/2076-2615/10/8/1392/s1, Figure S1: Effect of digestible glycine equivalent ( $\mathrm{dGly}_{\text {equi }}$ ) intake on average daily gain (ADG) of broiler chickens at varying digestible cysteine (dCys) and medium choline (Cho) intake from days 7 to 21 (a). The effect of dGlyequi intake on ADG of broiler chickens at varying Cho and low, medium, and high dCys intake from days 7 to $21(\mathbf{b}-\mathbf{d})$. dCys and Cho intake levels correspond to level $-1,0$, and +1 based on the fractional composite design. Figure S2: Effect of digestible glycine equivalent (dGly equi) intake on average daily feed intake (ADFI) of broiler chickens at varying digestible cysteine (dCys) and medium choline (Cho) intake from days 7 to 21 (a) and effects of $\mathrm{dGly}_{\text {equi }}$ intake on ADFI of broiler chickens at varying Cho and low, medium, and high dCys intake from days 7 to $21(\mathbf{b}-\mathbf{d})$. dCys and Cho intake levels correspond to level $-1,0$, and +1 based on the fractional composite design. Table S1: Analyzed concentrations of nutrients other than glycine equivalent, cysteine, and choline of the experimental diets $(\mathrm{g} / \mathrm{kg}$ on a $88 \%$ dry matter basis unless otherwise stated). Table S2: Neural network model equations for gain:feed ratio, excreta characteristics, and blood metabolites. Table S3: Description of neural network models for the prediction of traits that were not further described because $R^{2}$ was $\leq 0.50$ or because model results were difficult to interpret biologically.

Author Contributions: Conceptualization, P.H., W.S. and M.R.; methodology, P.H., W.S., H.A., J.K. and M.R.; software, H.A. and J.K.; validation, W.S. and M.N.; formal analysis, P.H., H.A., J.K. and M.N.; investigation, P.H., W.S., J.K. and M.N.; resources, H.A., V.D.N. and M.R.; data curation, P.H. and H.A.; writing-original draft preparation, P.H.; writing-review and editing, W.S., H.A., J.K., M.N., V.D.N. and M.R.; visualization, P.H. and W.S.; supervision, W.S. and M.R.; project administration, W.S. and M.R.; funding acquisition, M.R. All authors have read and agreed to the published version of the manuscript.

Funding: This research was funded by Evonik Nutrition \& Care GmbH.

Conflicts of Interest: V.D.N. is an employee of Evonik Nutrition \& Care GmbH. All other authors declare that they have no conflict of interest. The funders had no role in the design of the study; in the collection, analyses, or interpretation of data; in the writing of the manuscript, or in the decision to publish the results.

\section{References}

1. Dean, D.W.; Bidner, T.D.; Southern, L.L. Glycine supplementation to low protein, amino acid supplemented diets supports optimal performance of broiler chicks. Poult. Sci. 2006, 85, 288-296. [CrossRef] [PubMed]

2. Ospina-Rojas, I.C.; Murakami, A.E.; Eyng, C.; Nunes, R.V.; Duarte, C.R.A.; Vargas, M.D. Commercially available amino acid supplementation of low-protein diets for broiler chickens with different ratios of digestible glycine+serine:lysine. Poult. Sci. 2012, 91, 3148-3155. [CrossRef] [PubMed]

3. Awad, E.A.; Zulkifli, I.; Soleimani, A.F.; Loh, T.C. Individual non-essential amino acids fortification of a low-protein diet for broilers under the hot and humid tropical climate. Poult. Sci. 2015, 94, 2772-2777. [CrossRef] [PubMed]

4. Siegert, W.; Rodehutscord, M. The relevance of glycine and serine in poultry nutrition: A review. Br. Poult. Sci. 2019, 60, 579-588. [CrossRef] [PubMed]

5. Velíšek, J.; Cejpek, K. Biosynthesis of food constituents: Amino acids: 2. The alanine-valine-leucine, serine-cysteine-glycine, and aromatic and heterocyclic amino acids groups-a review. Czech J. Food Sci. 2006, 24, 45-58. [CrossRef]

6. Siegert, W.; Ahmadi, H.; Rodehutscord, M. Meta-analysis of the influence of dietary glycine and serine, with consideration of methionine and cysteine, on growth and feed conversion of broilers. Poult. Sci. 2015, 94, 1853-1863. [CrossRef]

7. Mackenzie, C.G.; Frisell, W.R. The metabolism of dimethylglycine by liver mitochondria. J. Biol. Chem. 1958, 232, 417-427.

8. Frontiera, M.S.; Stabler, S.P.; Kolhouse, J.F.; Allen, R.H. Regulation of methionine metabolism: Effects of nitrous oxide and excess dietary methionine. J. Nutr. Biochem. 1994, 5, 28-38. [CrossRef] 
9. Slow, S.; McGregor, D.O.; Lever, M.; Lee, M.B.; George, P.M.; Chambers, S.T. Dimethylglycine supplementation does not affect plasma homocysteine concentrations in pre-dialysis chronic renal failure patients. Clin. Biochem. 2004, 37, 974-976. [CrossRef]

10. Siegert, W.; Ahmadi, H.; Helmbrecht, A.; Rodehutscord, M. A quantitative study of the interactive effects of glycine and serine with threonine and choline on growth performance in broilers. Poult. Sci. 2015, 94, 1557-1568. [CrossRef]

11. Seegmiller, J.E. Purine metabolism. Arthritis Rheum. 1975, 18, 681-686. [CrossRef] [PubMed]

12. Box, G.E.P.; Hunter, W.G.; Hunter, J.S. Statistics for Experimenters: An Introduction to Design, Data Analysis and Model Building; Wiley: New York, NY, USA, 1978.

13. Gesellschaft für Ernährungsphysiologie. Energie- und Nährstoffbedarf landwirtschaftlicher Nutztiere. 7. Empfehlungen zur Energie - und Nährstoffversorgung der Legehennen und Masthühner (Broiler); DLG: Frankfurt am Main, Germany, 1999.

14. Hofmann, P.; Siegert, W.; Kenéz, Á.; Naranjo, V.D.; Rodehutscord, M. Very low crude protein and varying glycine concentrations in the diet affect growth performance, characteristics of nitrogen excretion, and the blood metabolome of broiler chickens. J. Nutr. 2019, 149, 1122-1132. [CrossRef] [PubMed]

15. Donsbough, A.L.; Powell, S.; Waguespack, A.; Bidner, T.D.; Southern, L.L. Uric acid, urea, and ammonia concentrations in serum and uric acid concentration in excreta as indicators of amino acid utilization in diets for broilers. Poult. Sci. 2010, 89, 287-294. [CrossRef] [PubMed]

16. Verband Deutscher Landwirtschaftlicher Untersuchungs- und Forschungsanstalten. Handbuch der landwirtschaftlichen Versuchs- und Untersuchungsmethodik (VDLUFA-Methodenbuch), Vol. III. Die chemische Untersuchung von Futtermitteln, 1st ed.; VDLUFA: Darmstadt, Germany, 2007.

17. Association of Official Analytical Chemists. Official Methods of Analysis of AOAC International, 16th ed.; AOAC International: Arlington, VA, USA, 1995.

18. Zeller, E.; Schollenberger, M.; Kühn, I.; Rodehutscord, M. Hydrolysis of phytate and formation of inositol phosphate isomers without or with supplemented phytases in different segments of the digestive tract of broilers. J. Nutr. Sci. 2015, 4, e1. [CrossRef] [PubMed]

19. Siegert, W.; Wild, K.J.; Schollenberger, M.; Helmbrecht, A.; Rodehutscord, M. Effect of glycine supplementation in low protein diets with amino acids from soy protein isolate or free amino acids on broiler growth and nitrogen utilisation. Br. Poult. Sci. 2016, 57, 424-434. [CrossRef] [PubMed]

20. Haupt, R.L.; Haupt, S.E. Practical Genetic Algorithms, 2nd ed.; Wiley: Hoboken, NJ, USA, 2004.

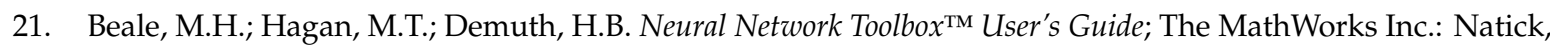
MA, USA, 2017.

22. Saltelli, A. Making best use of model evaluations to compute sensitivity indices. Comput. Phys. Commun. 2002, 145, 280-297. [CrossRef]

23. Goldstein, D.L.; Skadhauge, E. Renal and extrarenal regulation of body fluid composition. In Sturkie's Avian Physiology, 5th ed.; Whittow, G.C., Ed.; Academic Press: London, UK, 2000; pp. 265-297.

24. Bregendahl, K.; Sell, J.L.; Zimmerman, D.R. Effect of low-protein diets on growth performance and body composition of broiler chicks. Poult. Sci. 2002, 81, 1156-1167. [CrossRef]

25. Gomide, E.M.; Rodrigues, P.B.; Zangeronimo, M.G.; Bertechini, A.G.; dos Santos, L.M.; Alvarenga, R.R. Nitrogen, calcium and phosphorus balance of broilers fed diets with phytase and crystalline amino acids. Ciênc. Agrotec. 2011, 35, 591-597. [CrossRef]

26. Kriseldi, R.; Tillman, P.B.; Jiang, Z.; Dozier, W.A., III. Effects of feeding reduced crude protein diets on growth performance, nitrogen excretion, and plasma uric acid concentration of broiler chicks during the starter period. Poult. Sci. 2018, 97, 1614-1626. [CrossRef]

27. Rodehutscord, M.; Pack, M. Estimates of essential amino acid requirements from dose-response studies with rainbow trout and broiler chicken: Effect of mathematical model. Arch. Anim. Nutr. 1999, 52, $223-244$. [CrossRef]

28. Powell, S.; Bidner, T.D.; Southern, L.L. Effects of glycine supplementation at varying levels of methionine and cystine on the growth performance of broilers fed reduced crude protein diets. Poult. Sci. 2011, 90, 1023-1027. [CrossRef] [PubMed]

29. Stipanuk, M.H. Sulfur amino acid metabolism: Pathways for production and removal of homocysteine and cysteine. Annu. Rev. Nutr. 2004, 24, 539-577. [CrossRef] [PubMed] 
30. Karasawa, Y. Ammonia production from uric acid, urea, and amino acids and its absorption from the ceca of the cockerel. J. Exp. Zool. Suppl. 1989, 3, 75-80. [CrossRef] [PubMed]

31. Karasawa, Y.; Maeda, M. Role of caeca in the nitrogen nutrition of the chicken fed on a moderate protein diet or a low protein diet plus urea. Br. Poult. Sci. 1994, 35, 383-391. [CrossRef]

32. Pillai, P.B.; Fanatico, A.C.; Beers, K.W.; Blair, M.E.; Emmert, J.L. Homocysteine remethylation in young broilers fed varying levels of methionine, choline, and betaine. Poult. Sci. 2006, 85, 90-95. [CrossRef] [PubMed]

33. Pillai, P.B.; Fanatico, A.C.; Blair, M.E.; Emmert, J.L. Homocysteine remethylation in broilers fed surfeit choline or betaine and varying levels and sources of methionine from eight to twenty-two days of age. Poult. Sci. 2006, 85, 1729-1736. [CrossRef] [PubMed]

34. Dilger, R.N.; Garrow, T.A.; Baker, D.H. Betaine can partially spare choline in chicks but only when added to diets containing a minimal level of choline. J. Nutr. 2007, 137, 2224-2228. [CrossRef]

35. Emmert, J.L.; Garrow, T.A.; Baker, D.H. Hepatic betaine-homocysteine methyltransferase activity in the chicken is influenced by dietary intake of sulfur amino acids, choline and betaine. J. Nutr. 1996, 126, 2050-2058.

36. Emmert, J.L.; Webel, D.M.; Biehl, R.R.; Griffiths, M.A.; Garrow, L.S.; Garrow, T.A.; Baker, D.H. Hepatic and renal betaine-homocysteine methyltransferase activity in pigs as affected by dietary intakes of sulfur amino acids, choline, and betaine. J. Anim. Sci. 1998, 76, 606-610. [CrossRef]

37. Finkelstein, J.D.; Martin, J.J.; Harris, B.J.; Kyle, W.E. Regulation of hepatic betaine-homocysteine methyltransferase by dietary betaine. J. Nutr. 1983, 113, 519-521. [CrossRef]

38. Patience, J.F. A review of the role of acid-base balance in amino acid nutrition. J. Anim. Sci. 1990, 68, 398-408. [CrossRef] [PubMed]

39. Hamm, L.L.; Simon, E.E. Roles and mechanisms of urinary buffer excretion. Am. J. Physiol. Renal Physiol. 1987, 253, F595-F605. [CrossRef] [PubMed]

40. Coon, C.; Balling, R. Asparagine and glutamine metabolism in chicks. Poult. Sci. 1984, 63, 717-729. [CrossRef] [PubMed]

41. Borges, S.A.; Fischer da Silva, A.V.; Ariki, J.; Hooge, D.M.; Cummings, K.R. Dietary electrolyte balance for broiler chickens under moderately high ambient temperatures and relative humidities. Poult. Sci. 2003, 82, 301-308. [CrossRef]

42. Ahmadi, H.; Golian, A. Response surface and neural network models for performance of broiler chicks fed diets varying in digestible protein and critical amino acids from 11 to 17 days of age. Poult. Sci. 2011, 90, 2085-2096. [CrossRef]

43. Arifovic, J.; Gencay, R. Using genetic algorithms to select architecture of a feedforward artificial neural network. Phys. A 2001, 289, 574-594. [CrossRef]

44. Elsken, T.; Metzen, J.H.; Hutter, F. Neural architecture search: A survey. J. Mach. Learn. Res. 2019, $20,1-21$.

45. Litzinger, S.; Klos, A.; Schiffmann, W. Compute-efficient neural network architecture optimization by a genetic algorithm. In Proceedings of the Artificial Neural Networks and Machine Learning-ICANN 2019: Deep Learning: 28th International Conference on Artificial Neural Networks, Munich, Germany, 17-19 September 2019, Part II, 1st ed.; Tetko, I., Kůrková, V., Karpov, P., Theis, F., Eds.; Springer: Cham, Switzerland, 2019; pp. 387-392.

(C) 2020 by the authors. Licensee MDPI, Basel, Switzerland. This article is an open access article distributed under the terms and conditions of the Creative Commons Attribution (CC BY) license (http://creativecommons.org/licenses/by/4.0/). 